\section{RADAR CONTINUED}

\section{Radar Techniques for Detection, Tracking and Navi- gation}

Edited by W. T. Blackband. (Proceedings of the 8th Symposium of the AGARD Avionics Panel, London, September 21-25, 1964.) Pp. viii +608. (New York and London : Gordon and Breach, 1966. Distributed in the U.K. by Blackie and Sons, Ltd.) $\$ 37 \cdot 50$.

ONE of the useful organizations which has stemmed from NATO has been the Advisory Group on Aeronautical Research and Development (AGARD). This organization covers the usual fields of aeronautical research including aerodynamies and propulsion, and also includes in its activities that broad field of ancillary devices which is loosely termed "Avionics". AGARD takes the realistic view that electronic devices are essential elements of any airborne vehicle whether it is to be used for military or civil purposes.

Over the years AGARD has held a number of symposia which have been devoted to various aspects of the avionic field, and the present volume covers such a meeting which was held in September 1964 and which was devoted to the themes of radar techniques for detection, tracking and navigation. This volume consists of thirty-one papers which have been written by engineers and scientists active in the field of radar who are working in government, university or industrial research laboratories located in the various NATO countries.

Clutter is still the principal enemy of radar system designers and a number of papers in the present volume are concerned with the modulation problem and of how radar systems can be devised which will provide methods of enhancing the probability of detecting moving targets in the presence of clutter. The technique of pulse compression is described; frequency modulation is employed within the pulse together with a dispersive line in the receiver system which provides time delay varying with the frequency. In this way the effective pulse length of the radar can be compressed to $1 / 1000$ th of the transmitted pulse and resolution improved accordingly.

A number of the authors recognize that the target itself can materially affect the nature of the reflected signal. This interaction may prejudice radar detection on the one hand, but it can also provide information about the target which can be extracted by appropriate signal processing. This kind of study has been applied to the examination of the radar echoes from the planets, designated somewhat quaintly as "unco-operative targets", the non-co-operation arising from the remoteness of these bodies and the nature of their reflecting surfaces. It is very impressive to read that the power density of the radar echo from Venus at its apogee is $3 \times 10^{-25} \mathrm{~W}$. Radar echoes from extended scatterers have also been considerod by a number of authors, and these papers include careful examination of the nature of ground returns, Dopplor navigation, ground mapping and also the applications of radar to meteorology.

Although a number of the papers deal with the advanced theory of radar systems and the nature of radar information, the techniques of radar are not neglected, and improvements in transmitters, frequency and phase scanning arrays are dealt with.

It is probably true to say that even during the war years the main difficulty was not so much the collection of radar information as the ability to analyse and present the information contained in the echo signals. Some of the papers show very clearly that the omergence of a new electronics, based on solid state devices, is providing the means for the storage and analysis of radar information on a scale, and with a wealth of detail, which greatly transcends anything that was possible during the war. The application of computers operated in the real time mode permits executive commands to be extracted from this body of information and used for the purpose of aircraft control in both the civil and military case.

It is clear from this volume that radar research and development is still very active indeed, and that methods of radar monitoring the air space have become most subtle. Although the papers in a collection such as this are bound to be somewhat uneven, they never fail to be exciting and interesting, and the book may be taken as a good presentation of radar developments which have taken place during the past ten years. The complexity of radar equipment is now so great, and the time scale of development so long, that although two years have passed since the AGARD conference that prompted these papers the book is still up to date and will be of great value to radar research men for some years to come.

E. EASTWOOD

\section{SINGULAR WAVES}

\section{The Plane Wave Spectrum Representation of Electro- magnetic Fields}

By P. C. Clemmow. (International Series of Monographs in Electromagnetic Waves, Vol. 12.) Pp. vii 184. (Oxford, London and New York: Pergamon Press, Ltd., 1966.) 50s. net.

IN 1919 Weyl published the formula

$$
2 \pi \frac{\exp \{i k R\}}{i k R}=\int_{0}^{2 \pi} \mathrm{d} \varphi \int_{L} \exp \{i k(\alpha x+\beta y+\gamma z)\} \sin \theta d \theta
$$

valid for $z>0, \varphi, \theta$ being the polar angles of the unit vector $(\alpha, \beta, \gamma)$ referred to the $z$-axis, $R^{2}=x^{2}+y^{2}+z^{2}$, and $L$ a certain path of integration in the complex $\theta$. plane. He thereby obtained the plane wave representation of a singular solution of the wave equation in the presence of a point source at the co-ordinate origin. The superposition involves inhomogeneous plane waves or alternatively plane waves travelling along imaginary directions, and Weyl's step is crucial in obtaining an expansion or representation theorem involving plane waves for general electromagnetic fields generated by sources or bounded by surfaces. This theorem received little attention-it is not mentioned in the older textbooks-until soon after the Second World War when Brooker and Clemmow took up the idea. In two classic papers they demonstrated the application of the angular spectrum of plane waves to radio propagation over flat surfaces, to certain aerial problems and to diffraction theory. Soon afterwards, Clemmow obtained the exact solution of a wide class of two-dimensional diffraction problems and there have been other applications since then.

Dr. Clemmow has now written a connected introduction to the subject. It is a most beautiful book and will surely become a classic in its field. Maxwell's equations in M.K.s. units and the idea of induced electric and magnetic surface current densities are of course assumed to be familiar, and the reader must be at home in the complex plane, but that is all that is required. A self-contained and careful exposition establishes, for example, the plane wave representation, its relation to other representations and the way to separate off the field in the radiation zone. Then the applications are considered. These cover diffraction by a plane screen, propagation over plane surfaces, the fields of point particles, both synchrotron radiation and the Cerenkov radiation and finally sources in anisotropic media. There is a very satisfying demonstration of the optical theorem and Babinet's principle.

The text is completely self-contained; there is not a single reference to other material or authors, and Dr. Clemmow is too modest to mention his own contributions to the subject. He clearly enjoyed writing the book, and I enjoyed reading it. It can be most warmly recommended, not only to students of electromagnetic theory but to everybody concerned with scattering problems. S. ZIENAU 